\title{
Nanostructure engineered chemical sensors for hazardous gas and vapor detection
}

\author{
Jing $\mathrm{Li}^{*}$ and Yijiang $\mathrm{Lu}$ \\ Center for Nanotechnology, NASA Ames Research Center, Moffett Field, CA 94035
}

\begin{abstract}
A nanosensor technology has been developed using nanostructures, such as single walled carbon nanotubes (SWNTs) and metal oxides nanowires or nanobelts, on a pair of interdigitated electrodes (IDE) processed with a siliconbased microfabrication and micromachining technique. The IDE fingers were fabricated using thin film metallization techniques. Both in-situ growth of nanostructure materials and casting of the nanostructure dispersions were used to make chemical sensing devices. These sensors have been exposed to hazardous gases and vapors, such as acetone, benzene, chlorine, and ammonia in the concentration range of $\mathrm{ppm}$ to $\mathrm{ppb}$ at room temperature. The electronic molecular sensing in our sensor platform can be understood by electron modulation between the nanostructure engineered device and gas molecules. As a result of the electron modulation, the conductance of nanodevice will change. Due to the large surface area, low surface energy barrier and high thermal and mechanical stability, nanostructured chemical sensors potentially can offer higher sensitivity, lower power consumption and better robustness than the state-of-the-art systems, which make them more attractive for defense and space applications. Combined with MEMS technology, light weight and compact size sensors can be made in wafer scale with low cost.
\end{abstract}

Keywords: Nanostructures, nanosensors, chemical sensing devices, carbon nanotubes, gas and vapor detection

\section{INTRODUCTION}

Nanotechnology deals with materials, devices, and systems whose structures and components exhibit novel and significantly improved physical, chemical and biological properties due to their nanoscale size. As one class of nanostructured materials, carbon nanotubes (CNTs) have been receiving much attention due to their remarkable mechanical properties and unique electronic properties as well as the high thermal and chemical stability and excellent heat conduction. Carbon nanotubes have a large surface to volume ratio and aspect ratio with the diameter of a few $\mathrm{nm}$ and length up to $100 \mathrm{~m}$. This extremely thin wire, a unique one in the carbon family, possesses hardness like diamond and conductivity like graphite. Since the electronic property of carbon nanotubes is a strong function of their atomic structure and mechanical deformations, such relationships make them useful to develop extremely small sensors that are sensitive to the chemical environments [1].

In the past few years, CNT sensors have been demonstrated with improved performance compared to conventional sensor utilizing materials such as semiconducting metal oxides thin films [2], silicon [3], organic/polymeric materials [4], and carbon black-polymer composites [5] This paper focuses on the carbon nanotube sensors and addresses recent developments in CNT based chemical sensors and their performance compared with conventional chemical sensors on the market.

Carbon nanotubes have been used as is and have also been modified with polymer coatings and catalytic metal clusters for sensor applications in our lab. These sensors have been exposed to nitrogen dioxide, acetone, benzene, nitrotoluene, chlorine, and ammonia in the concentration range of ppm to $\mathrm{ppb}$ at room temperature with a good reproducibility from sensor to sensor.

Advances in chemical sensor technology are continuously needed for improved sensitivity and fast response to extract more accurate and precise information from changes in the environment. The working principle of these sensors and future possible real world sensing applications are discussed.

\footnotetext{
•Corresponding author: jingli@mail.arc.nasa.gov; phone 1-650-604-4352; fax 1-650-604-5244
} 


\section{NANOSENSORS FABRICATION}

A single carbon nanotube field effect transistor was fabricated as an electronic component by a group at IBM [6], and Dekker at Delft [7]. Soh [8] and Dai [9] have combined the synthesis of the carbon nanotube and the microfabrication of the electrodes on a silicon (Si) substrate that is used as a back gate. There are four steps, with electron beam lithography using polymethylmethacrylate as the resist, involved in this device fabrication process: 1) patterning of the electrodes windows ( $5 \mathrm{~m} \times 5 \mathrm{~m})$ using alignment marks on the Si substrate; 2 ) forming the catalyst islands in the electrode windows for carbon nanotube growth; 3 ) growing the carbon nanotubes via methane CVD to bridge the islands; 4 ) patterning and depositing the Ti/Au over the catalyst islands for electrical contact pads. The operation of these FETs was found to depend heavily on the ambient conditions, thus leading to the development of the nanotube FET for sensing applications. Since then, these have been demonstrations of chemical sensing of $\mathrm{NO}_{2}$, ammonia etc. [10]. While a single SWNT retains the advantages of sensitivity expected from a nanotube, fabrication of a CNT FET is relatively complex; it is particularly tedious to grow one semiconducting SWNT by CVD to bridge the contacts and the yield is poor.

Another way of making a sensing device with carbon nanotubes is to grow the nanotubes

somewhere else first, and then place them onto a device. Li and colleagues [11] have developed a gas sensor based on an interdigitated electrode (IDE) design with carbon nanotubes forming a network type of thin layer laid across the electrodes (see Fig. 1a). The conductivity of the CNT network changes upon exposure of dry air and dry air containing the gas or vapor [11]. The device fabrication process involved two simple steps: 1) making interdigitated electrodes ( $10 \mu \mathrm{m}$ of finger width and $8 \mu \mathrm{m}$ of gap between fingers) of $\mathrm{Ti} / \mathrm{Au}$ on silicon substrate using conventional lithography; 2) dispersing the purified carbon nanotubes [12] in a solvent and dispense a droplet onto the IDE area (see figure 1a).

A

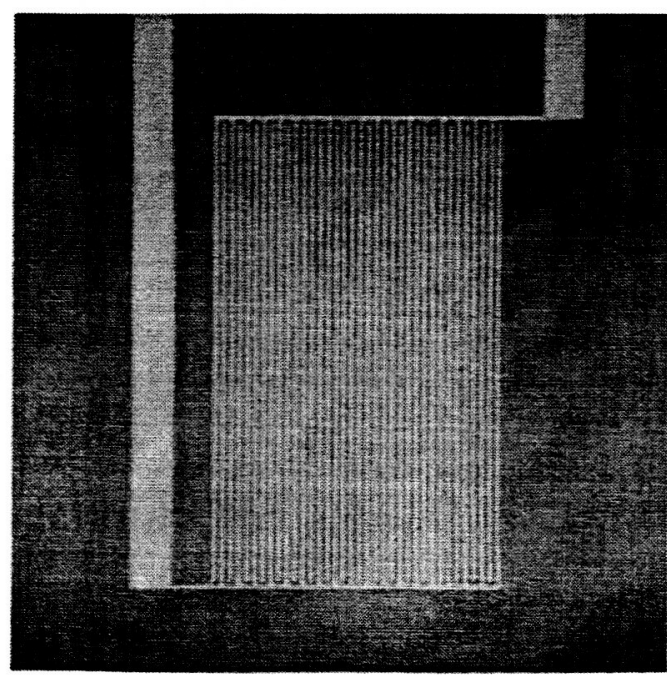

B

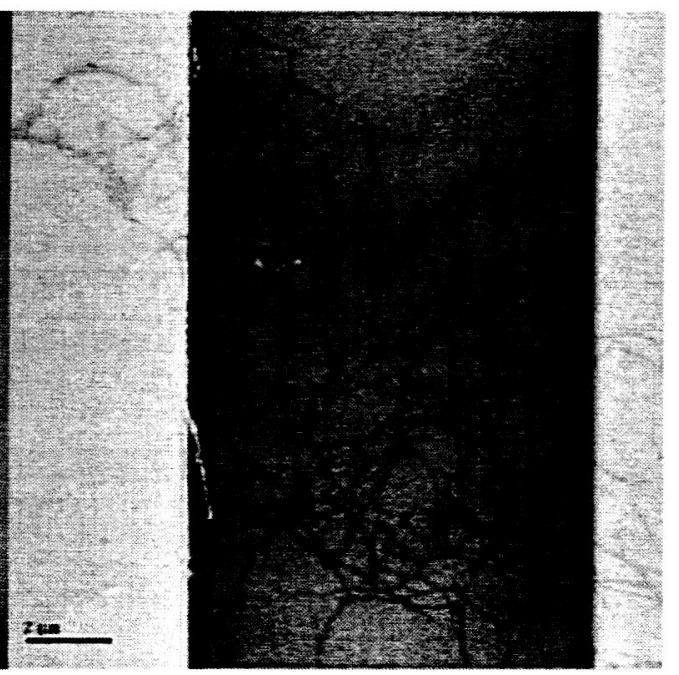

Fig 1 SWNTs across the fingers of an interdigitated electrode: A) Photo image of an IDE; B) SEM image of SWNTs across two gold electrodes.

The sensor performance can be tuned by varying the IDE finger gap size, and the density of the carbon annotate across the electrodes by varying the concentration of the carbon annotate dispersion. This is a simple process with a high sensor yield, which can be scaled up for mass production. This technology developed at NASA Ames Research Center has attracted much of attention from industry and Integrated Nanosystems, Inc. is commercializing this technology for a variety of applications. 
There are several other deposition methods useful in sensor fabrication such as spin-coating, ink jetting, dipping-coating, and air brushing. These methods can easily provide carbon nanotube sensors with different chemical functionalities forming a sensor array. Carbon nanotubes can be pretreated by several methods: 1) modifying the surface of the nanotubes with functional groups, such as carboxylic group for base molecules, ammine groups for acidic molecules and other aromatic groups for large organic molecules; 2) Doping the carbon nanotubes with catalytic metal clusters such as Pd, Pt and $\mathrm{Ag}$ for hydrogen and hydrocarbon detection, and $\mathrm{Cu}$ and $\mathrm{Rh}$ for detecting nitric compounds. In this case, the carbon nanotube ensemble will act as a matrix that holds the metal binding sites for chemical sensing; 3) Coating the surface of carbon nanotubes with different polymers - such as polyethylene chlorosulfonated, hydroxypropyl cellulose, etc. used in commercial polymer based chemical sensing array for toxic gas, such as chlorine and hydrogen chloride, and organic vapor detection - that provides specific interactions with chemical species of interest.

The same approach of putting down the carbon nanotubes has been used by Collins [13] to form a gas sensor using the thin film of single walled carbon nanotube (SWNTs) deposited by its dispersion in dichloroethane; by Sumanasekera [14] to fabricate gas sensors for detection of hydrocarbons; by Chopra [15] and Ong [16] for making resonant-circuit sensors using multiwalled carbon nanotubes (MWNTs) for ammonia and carbon dioxide detection.

\section{SENSING EFFECTS IN CARBON NANOTUBES BASED SENSORS}

In this paper, the sensing platform is built based on the electrochemical type of mechanism. Electrochemistry implies the transfer of charge from one electrode to another electrode. This means that at least two electrodes constitute an electrochemical cell to form a closed electrical circuit. Due to the curvature of graphene sheet in nanotubes, the electron clouds change from a uniform distribution around the C-C backbone in graphite to an asymmetric distribution inside and outside the cylindrical sheet of the nanotube [17]. Since the electron clouds are distorted, a rich-electron conjugation formed outside the tube, therefore, makes the carbon nanotube electrochemically active at a certain level. Depending upon the electronegativity of both the carbon nanotubes and the gas molecules, either transfer electrons to or withdraw electrons from SWNTs possibly occur, thereby giving SWNTs more charge carriers or holes, respectively, which increases or decreases the SWNT conductance [18, 14]. Typical electrochemical interaction may be denoted as:

$$
\mathrm{CNT}+\mathrm{Gas}-\mathrm{CNT}^{\delta e} \mathrm{Gas}^{\delta+} \text { or }^{\mathrm{CNT}}{ }^{\delta+} \mathrm{Gas}^{\delta e}
$$

Where, $\delta$ is a number that indicates the amount of charge transferred during the interaction. By measuring the conductivity change of the CNT device, the concentration of the chemical species, such as gas molecules, can be measured. Another mechanism can also be used to explain the observed sensing signal, which is the Schottky effect by gas molecules intacted at the metal and carbon nanotube contact [19]. This Shottky effect mechanism sometimes is used to explain the sensing signal from those molecules that were calculated with mostly electrostatic in nature and a very little charger transfer with SWNT, such as ammonia [20]. The nanosensor may have both mechanisms coexist for its chemical sensing. One mechanism may be the dominating factor for the sensing signal over the other mechanism. The domination of which mechanism to the sensing signal is analyte dependent based upon its physiochemical properties, such as electronegativity and polarity, and is also contact metal dependent based on the work function match between the metal and the nanostructures (e.g. SWNTs).

\subsection{Pure carbon nanotubes}

The assembled interdigitated SWNT devices have been exposed to nitrogen dioxide $\left(\mathrm{NO}_{2}\right)$, ammonia, acetone, benzene, and nitrotoluene. The results for $\mathrm{NO}_{2}$, a molecule that has been extensively studied for nanotube sensors, and nitrotoluene, an organic vapor with nitrogen oxide group in benzene have been discussed in detail in ref. [11]. Here we show the results of sensor response to benzene (see figure 2) and acetone (see figure 3) for the demonstration of the SWNT sensors. The SWNT sensors responded to 20ppm and 50ppm benzene with clear sensing signal. The sensor has to be pretreated with UV light in order to detect acetone. The blue line shows the sensor response of SWNT sensor without UV light pretreatment to $1 \mathrm{ppm}$ and $10 \mathrm{ppm}$ acetone and the purple line shows clearly that the same sensor treated with UV light can detect $1 \mathrm{ppm}$ acetone easily (see figure 3 ). Three replicates of SWNT sensors were exposed to ammonia at different concentrations (see figure 4). The response curves of these three sensors show very reproducible results from 
one to the other. It gives a step function relation with the concentration of ammonia. However, the sensor response direction is opposite to that of $\mathrm{NO}_{2}$. This result shows there is some degree of selectivity from carbon nanotube itself due to the nature of different gas species.

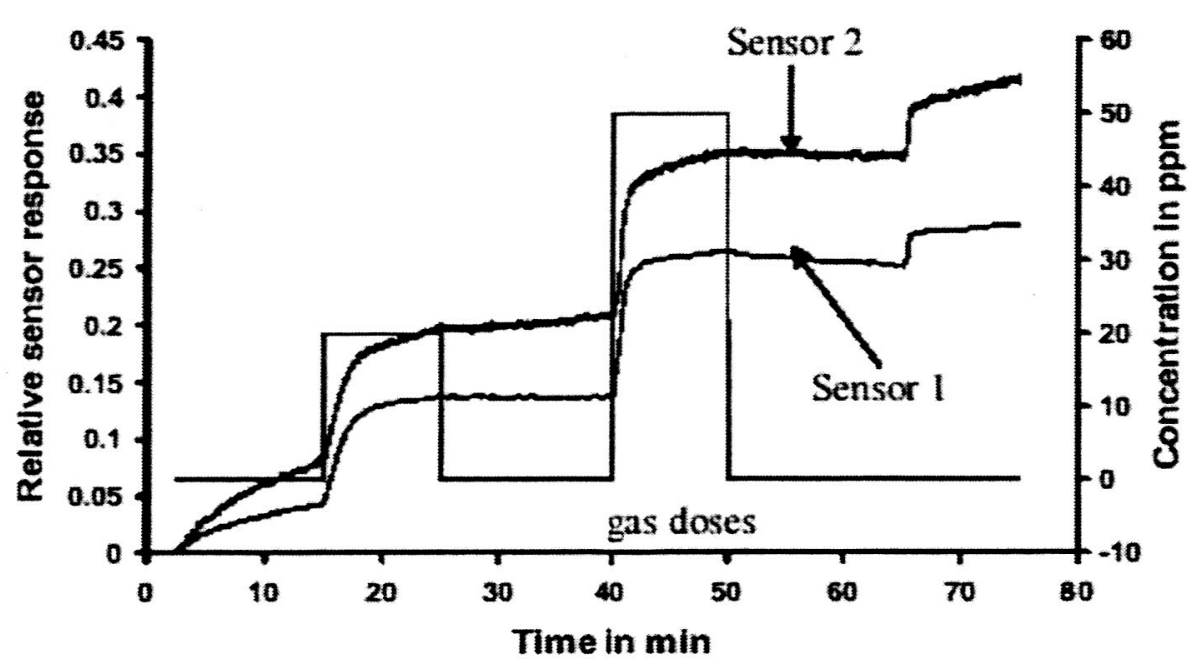

Fig 2. Two SWNT sensors exposed to benzene at 20ppm and 50ppm

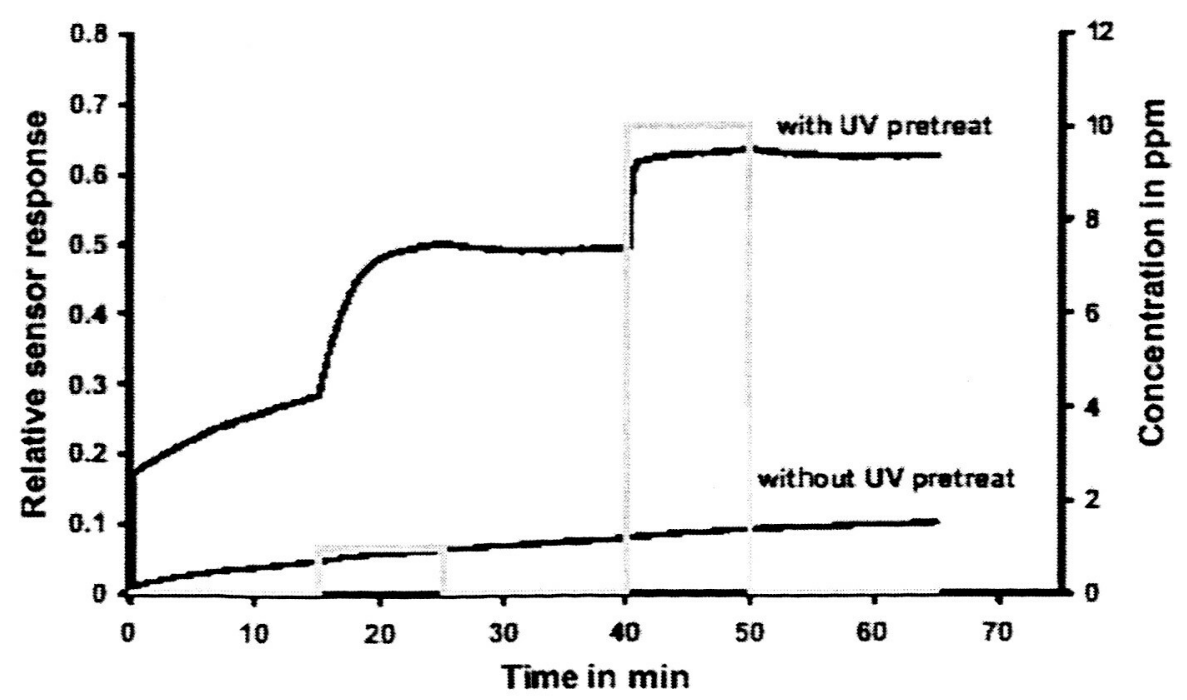

Fig 3. SWNT sensor exposed to acetone at $1 \mathrm{ppm}$ and $10 \mathrm{ppm}$ with and w/o UV pretreatment 


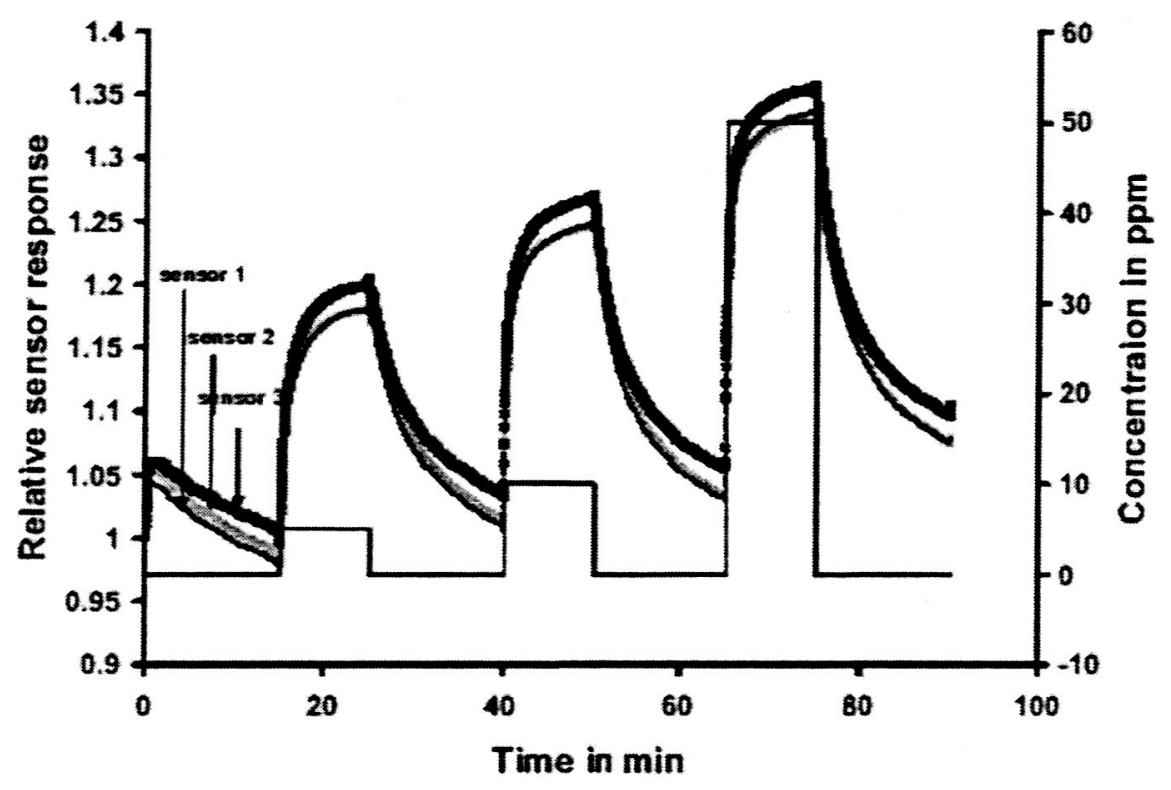

Fig 4. Three replicates of SWNT sensors respond to ammonia at 5ppm, 10ppm, 50ppm

The electronic molecular sensing in our SWNTs sensor platform can be understood by intra- and inter-tube interaction and contact barrier modulation in terms of electron modulation mechanisms. As a result of the partial charge transfer or contact barrier modulation, the conductance of p-type or hole-richer SWNTs will change. The relative change in resistance, before and during the gas and vapor exposures, is the sensor response signal. Plotting the sensor responses vs. the concentration of the gas and vapors, calibration curves can be obtained for later unknown gas and its concentration detection.

\subsection{Carbon nanotubes loaded with nano metal clusters}

The SWNTs have also been loaded with palladium nanoparticles (10-20 nm in diameter, see figure 5) for methane detection in ppm range at room temperature. Palladium (Pd) is known as catalyst for hydrogen and hydrocarbons. Both theory and experiment confirm the presence of long range, weakly bound complex $\mathrm{Pd}^{\delta+}\left(\mathrm{CH}_{4}\right)^{\delta-}$ even at room temperature [21]. This suggests that palladium interacted with the methane molecule to distort its hydrogen-carbon bond; therefore, it caused a partial charge transfer between methane molecules and Pd particles, which changes the conductance of the Pd-SWNTs matrix; hence, changes the current through it, providing a rapid, simple means of detection. Moreover, the gas molecules can be driven from the Pd-SWNTs sensing material using ultraviolet light and high temperatures, cleaning the sensor for further use. The detection of methane was demonstrated in the concentration range of 6-100 ppm in air (see figure 6a).

The sensor response is related to the logarithmic concentration of methane in air at room temperature (see figure $6 \mathrm{~b}$ ). The increase in temperature drives the larger sensor response and shorter response time. 


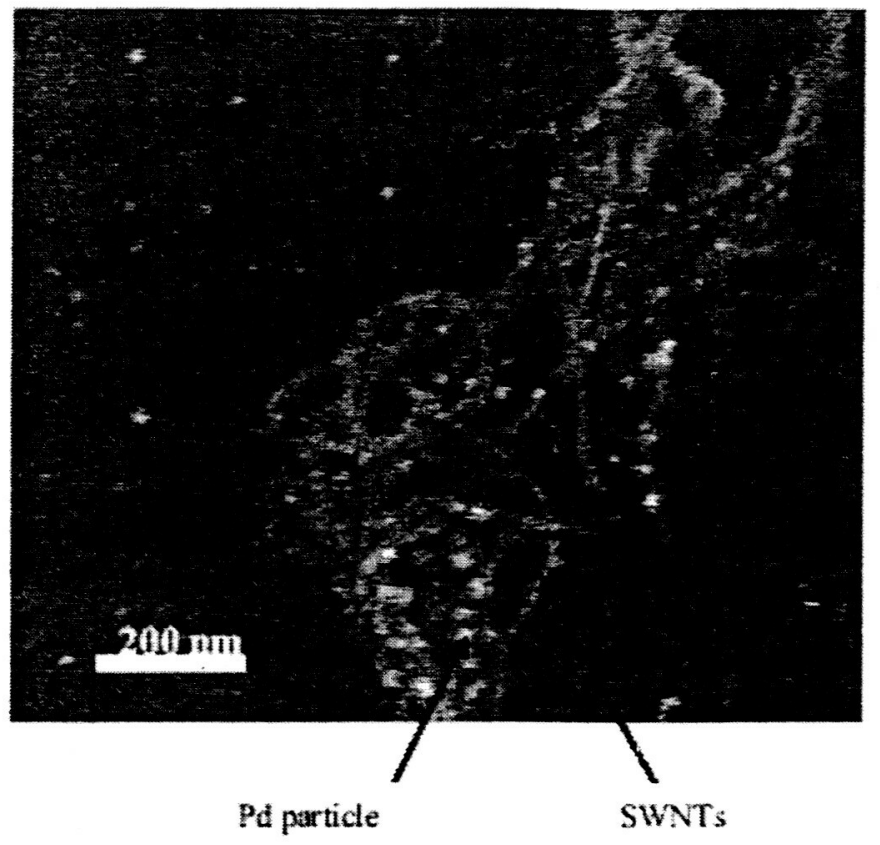

Fig 5 SEM image of SWNT bundles with Palladium nanoparticles loaded via CVD process

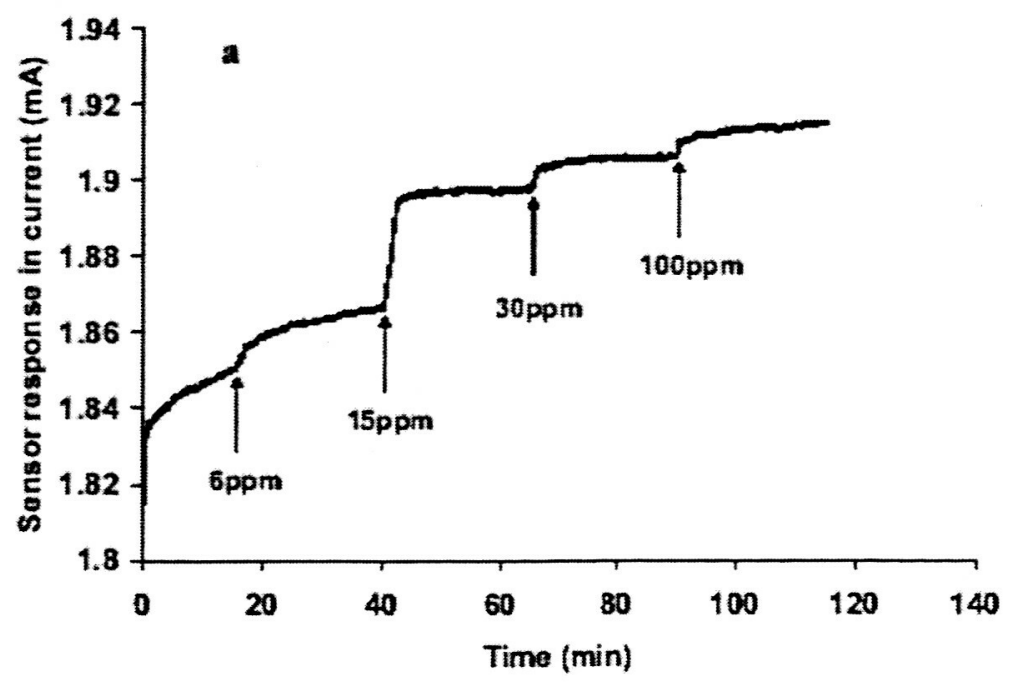




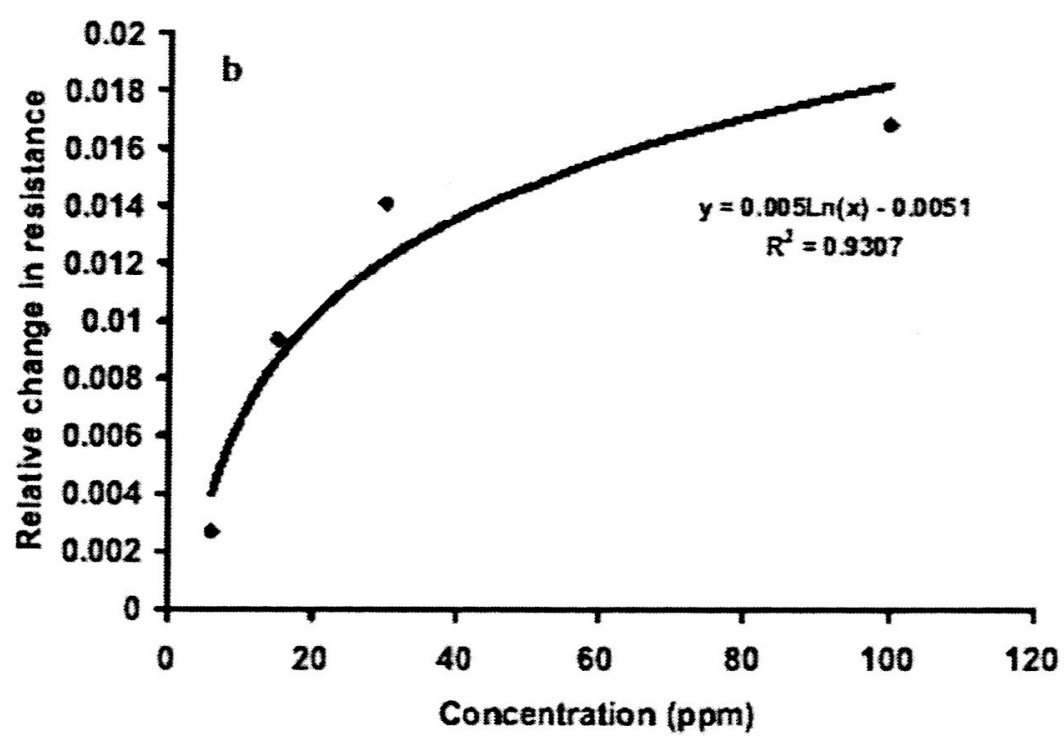

Fig 6. SWNTs-Pd sensor response to methane (a) and the calibration curve (b)

In this case of methane, Pd interacts with the methane trapped/adsorbed in the SWNTs. As the Pd catalyzes the methane to partial decomposition or distorts the $\mathrm{C}-\mathrm{H}$ bond, it partially donates electrons to one of the hydrogen atoms in the methane, causing the metallic Pd to lose partial electrons and become partially ionic palladium $\left(\mathrm{Pd}^{\delta \dagger}\right)$. The positively charged palladium particles/clusters in the SWNTs matrix tend to draw electrons from SWNTs which has pi-electrons rich outside of the tube. Carbon nanotubes are directly connected to the metal electrodes. Therefore, the changes in its conductivity before and after the methane exposure are measured. This is another type of mechanism with that the SWNTs act as a media for electrons transfer from SWNTs to facilitate formation of the complex of $\mathrm{Pd}-\mathrm{HCH}_{3}[21]$ and leave more or less holes in SWNTs. When the gas molecules are removed from the SWNTs, the Pd is recovered to metallic Pd and hence the conductance changes back to its initial level.

\subsection{Carbon nanotubes coated with polymers}

Carbon nanotubes do not sense all gases and vapors due to the chemical and physical properties of $\mathrm{CNT}$ and the detected gas molecules. Carbon nanotubes coated with different polymers - such as polyethylene chlorosulfonated, hydroxypropyl cellulose, polystyrene, polyvinylalcohol, etc. used in commercial polymer based chemical sensing array for organic vapor detection - that provides specific interactions with chemical species of interest. As this chemical treatment aims to get a specific interaction between the carbon nanotube matrix and gas molecules, it can improve the selectivity while maintaining the high SWNT sensor sensitivity.

Several polymer coated carbon nanotube sensors have been tested to different toxic gases, such as chlorine, hydrogen chloride. Carbon nanotubes coated with polyethylene (chlorosulfonated) provide highest sensitivity to chlorine (see figure 7) and hydroxypropyl cellulose was coated on carbon nanotubes for highest sensitivity to hydrochloric acid gas detection among the other polymers (see figure 8). 


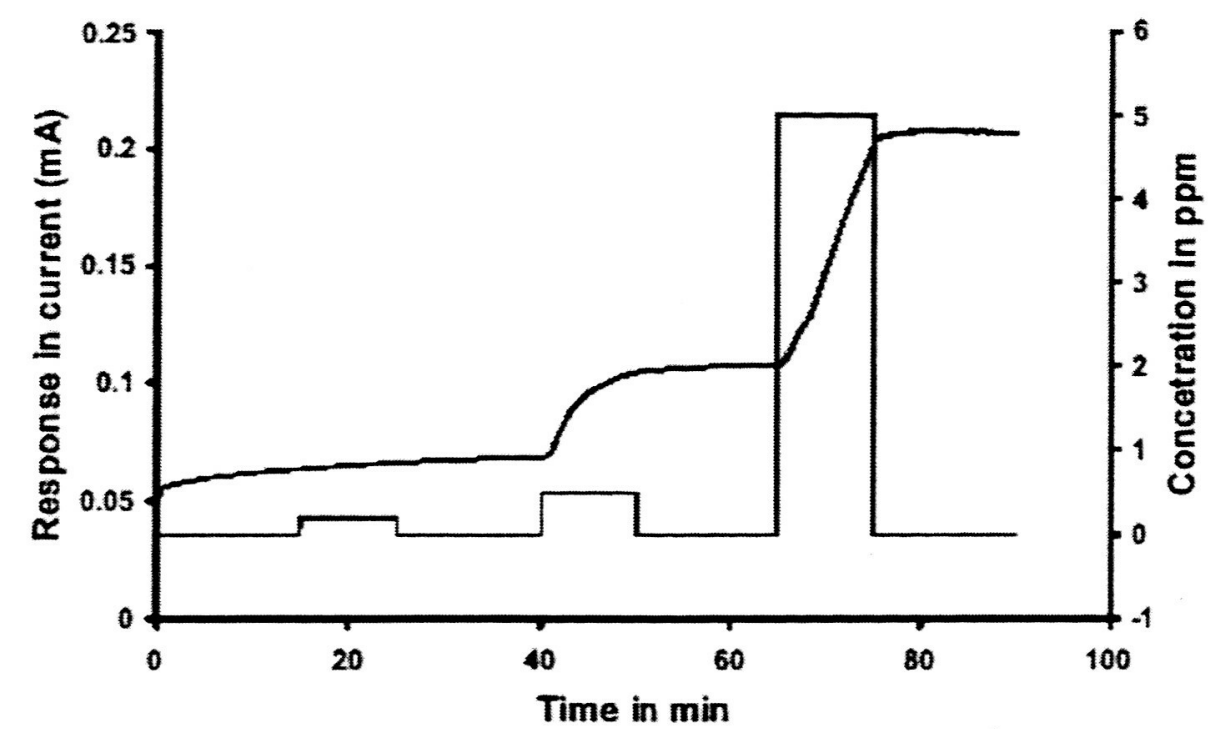

Fig 7. Polyethylene chlorosulfonated polymer coated SWNTs sensor for $\mathrm{Cl} 2$ detection

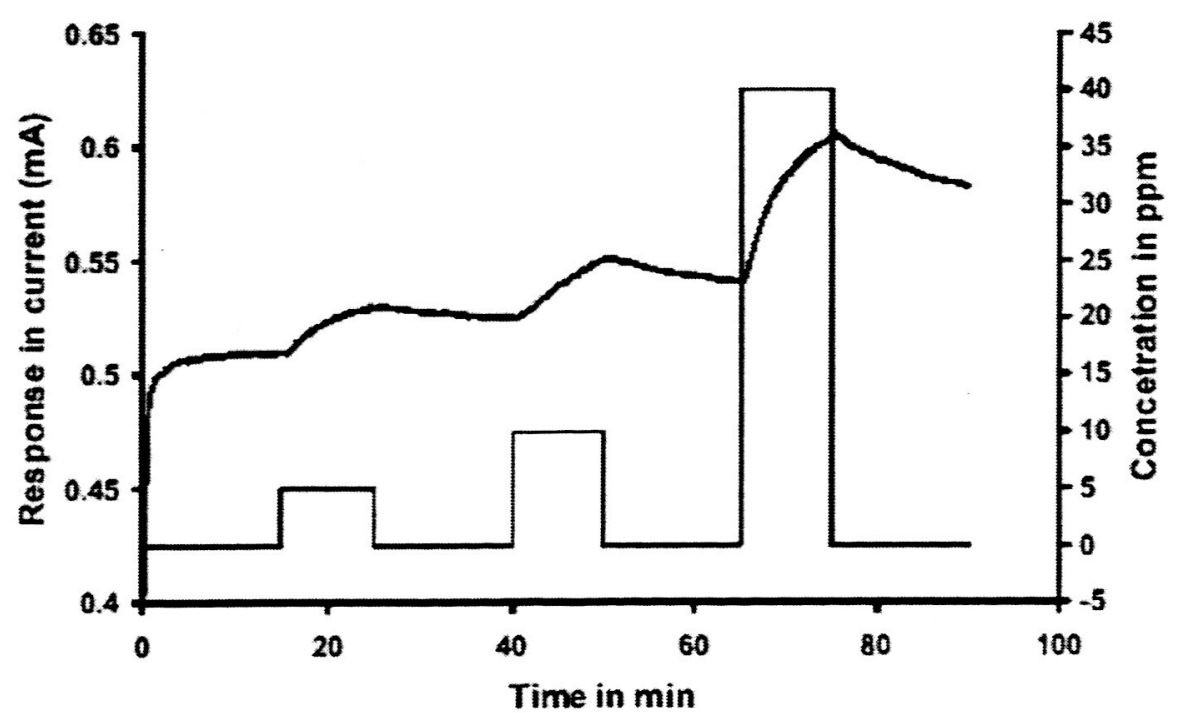

Fig 8. Hydroxypropyl œllulose coated SWNTs sensor for $\mathrm{HCl}$ detection

\section{COMPARISON OF NANOSENSOR AND CONVENTIONAL SENSORS}

Conventional sensors available currently include polymer sensors, metal oxides sensors, porous metal sensors (or catalytic beads), and electrochemical cells. Each type of sensor has its own advantages and disadvantages. Polymer sensors can operate at room temperature, but they are limited to low vapor pressure and large molecular weight gases and vapors. Both metal oxides and catalytic beads require high operating temperature $\left(350-600^{\circ} \mathrm{C}\right)$ can consume high power. The catalytic beads based sensors require typically $550^{\circ} \mathrm{C}$ for methane detection $[20,22]$. The heat of combustion in the presence of a gas is balanced by a reduction in electric heating power. Even with the nanostructured Pd (less than $10 \mathrm{~nm}$ particle size), the temperature for methane detection based on combustion would still be around $500^{\circ} \mathrm{C}$ while the sensitivity can be increased from the detection limit of $0.5 \%$ to $1 \%$ for conventional sensors to the $0.125 \%$ or below for 
nanoparticles [23] corresponding to the hundreds of ppm methane. Metal oxides sensors are usually for detection of small molecule gases in contrast to polymer sensors. Electrochemical cells are widely used for toxic gas detection. They require very low power, but there are only few electrochemical cells for limited type of gases that either are strong electron donor or withdrawer, which can cause an obvious electrode reaction.

Sensors using carbon nanotubes have demonstrated many benefits over commercial available sensors. Since every carbon atom is located on the surface of the nanotube, the expected sensitivity is very high. Distorted electron distribution makes the carbon nanotube chemically active, so that carbon nanotube sensor can operate at room temperature to observe the electrical change corresponding to the exposure of gas molecules. The extremely large surface area of carbon nanotubes of $1600 \mathrm{~m}^{2} / \mathrm{g}$ [12] ensures high sensitivity, for example, the detection limit of an IDE designed nano sensor is $44 \mathrm{ppb}$ for $\mathrm{NO}_{2}$ and 252 for nitrotoluene. Our Pd-SWNT sensors detected 6ppm methane at room temperature compared with Pd nanoparticle sensor that detects $<0.125 \%(1250 \mathrm{ppm}$ to hundreds $\mathrm{ppm})$ methane at $300^{\circ} \mathrm{C}$. Additional benefit of the IDE sensor platform design is the extended detection capability to organic vapors such as benzene, acetone and nitrotoluene.

In summary, SWNT, Pd doped SWNT, and polymer coated sensors have been demonstrated for the detection of $\mathrm{NO}_{2}$, nitrotoluene, benzene, acetone, $\mathrm{NH}_{2}, \mathrm{Cl}_{2}$ and $\mathrm{HCl}$, and trace amounts of methane in air at room temperature, showing an order of magnitude improvement in size, sensitivity and two orders of magnitude improvement in power consumption over conventional sensors. These sensors give reproducible trends and consistently respond to heat, and UV light for speedy recovery.

\section{APPLICATIONS}

Sensor development using nanostructure engineered materials such as CNTs for hazardous gases and vapors is at its early stages. The potential in various sectors is outlined below. The challenges are common to all these sectors: inexpensive fabrication steps, sensor robustness, reliability, reproducibility, and system integration. This sensing platform can be used not only for hazardous gas and vapor detection, but also can be used for other industrial and medical applications as well.

\subsection{Industry}

Carbon nanotube based chemical sensors possess high sensitivity, small size and low power consumption, which can be used to quickly detect the gas leakage (methane, toluene, etc.) for safety inspection and map the source of contamination. It can also be used to verify incoming raw materials at the delivery point. The technology can significantly reduce the amount of time and money spent analyzing those materials in a lab, as well as reducing the amount of materials handling. Most changes in chemical processes can be reflected in the changing composition of the vapor phase surrounding or contained within the process. Thus, the vapor phase sensors enable the quick assessment of the chemical status of most industrial processes. Examples are found across many sectors including food processing (coffee roasting and fermentation), petrochemical (plastics manufacture and gasoline blending) and consumer products (detergents and deodorants). Much like vision inspection is used to assess the visual integrity (color, shape, size) of products, olfactory inspection assesses the chemical integrity (consistency, presence of contaminants).

\subsection{Environment}

Increasing awareness and new regulations for safety and emission control make environmental monitoring one of the most desired amongst the numerous industrial and civil applications for which the development of reliable solidstate gas sensors is demanded. Current methods for air quality control approved by the standards consist of analytical techniques, which need the use of very costly and bulky equipment. For applications in this arena, sensors that are able to selectively detect various gases at a concentration level of a few ppb and in the form of low-cost portable handheld devices for continuous in-situ monitoring are needed. With unique advantages of high sensitivity, small size and low power consumption, and strong mechanical and thermal stability, carbon nanotube based chemical sensors are best fit for this type of application. The example is that carbon nanotubes sensors have been demonstrate for environmental hazardous detection, such as ammonia, nitrogen dioxide, benzene, chlorine and hydrogen chloride in this paper. 


\subsection{Defense}

Chemical sensors are very focused for security and defense applications due to their portability and low power consumption. Carbon nanotube sensors potentially can offer higher sensitivity and lower power consumption than the state-of-the-art systems, which make them more attractive for defense applications. Some examples include monitoring filter breakthrough, personnel badge detectors, embedded suit hermiticity sensors and other applications. Additionally, a wireless capability with the sensor chip can be used for networked mobile and fixed-site detection and warning systems for military bases, facilities and battlefield areas.

\subsection{Medical/Bio}

It is believed that chemical sensors would provide physicians with a quicker and more accurate diagnostic tool. Applications could include obtaining objective information on the identity of certain chemical compounds in exhaled air and excreted urine or body fluids related to specific metabolic conditions, certain skin diseases or bacterial infections, such as those common to leg or burn wounds. Additionally, the chemical sensors may provide more accurate, real-time patient monitoring during anesthesia administration. 


\section{REFERENCES}

1. Li, J. Carbon Nanotubes: Science and Applications, Chapter 9, Editor: M. Meyyappan, CRC Press, Boca Raton, FL, USA, 2004.

2. Heiland, G., Sensors and Actuators, Vol. 2, 343, 1982.

3. Mandelis, A. and Chrisofides, C., Physics, Chemistry, and Technology of Solid State Gas Sensor Devices, Wiley, New York, 1993.

4. Wrighton, M. S., Science, Vol. 231, 32, 1986.

5. Longergan, M. C., Severin,E. J., Doleman, B. J., Beaber, S. A., Grubbs, R. H., and Lewis, N. S., Chem. Mater. Vol. 8, 2298, 1996.

6. Collins, P. G., Arnold, M.S., Avouris, P., Science, Vol. 292, 706, 2001.

7. Tans, S.J., Verschueren, A. R. M., Dekker, C., Nature, Vol. 393, 49, 1998.

8. Soh, H. T., Quate, C. F., Morpurgo, A. F., Marcus, C. M., Kong, J., and Dai, H., Appl. Phys. Lett., Vol. 75, $627,1999$.

9. Dai, H., Kong, J., Zhou, C., Franklin, N., Tombler, T., Cassell, A., Fan, S., and Chapline, M., J. Phys.

Chem. B, Vol. 103, 11246, 1999.

10. Kong, J., Franklin, N. R., Zhou, C., Chapline, M. G., Peng, S., Cho, K., Dai, H., Science, Vol. 287, 622, 2000.

11. Li, J., Lu, Y., Ye, Q., Cinke, M., Han, J., and Meyyappan, M., Nano Lett., Vol. 3, 929, 2003.

12. Cinke, M., Li, J., Chen, B., Cassell, A., Delzeit, L, Han, J., and Meyyappan, M., Chem. Phys. Lett. Vol. 365, 69, 2002.

13. Collins, P.G., Bradley, K., Ishigami, M., and Zettl, A., Science, Vol. 287, 1801, 2000.

14. Sumanasekera, G.U., Adu, C.K.W., Fang, S., Eklund, P.C., Phys. Rev. Lett. Vol. 85, 1096, 2000.

15. Chopra, S., Pham, A., Gaillard, J., Parker, A., Rao, A. M., Appl. Phys. Lett. Vol. 80, 4632, 2002.

16. Ong, K. G., Zeng, K., Grimes, G.A., IEEE sens. J. Vol. 2, 82, 2002.

17. Meyyappan, M., Srivastava, D., Handbook of Nanoscience, Engineering, and Technology, Chapter 18, CRC Press, 2003.

18. Adu, C.K.W., Sumanasekera, G.U., Pradhan, B.K., Romero, H.E., Eklund, P.C., Chem. Phys. Lett., Vol. 337, 31, 2001.

19. Heinze, S., Tersoff, J., Martel, R., Derycke, V., Appenzeller, J., Avouris, Ph., Phys. Rev. Lett, Vol. 85, $1710,2000$.

20. Bauschlicher, C.W. Jr., Ricca, A., Phys. Rev. B 70, 115409, 2004.

21. Campell, M. L. Chem. Phys. Lett. Vol. 365, 361, 2002.

Martin, S. J., Frye, G. C., and Ricco, A. J., Anal. Chem. Vol. 65, $2910,1993$.

22. Kohl, D, J. Phys. D: Appl. Phys. Vol. 34, 125, 2001.

23. Bartlett, P. N; Guerin, S. Anal. Chem. Vol. 75, 126, 2003. 